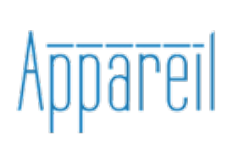

Appareil

$22 \mid 2020$

Une philosophie de l'appareil

\title{
Inscrire les fantômes dans les villes
}

Innerver les corps pour résister

\section{Adolfo Vera}

\section{(2) OpenEdition}

Journals

Édition électronique

URL : http://journals.openedition.org/appareil/3488

DOI : 10.4000/appareil.3488

ISSN : 2101-0714

Éditeur

MSH Paris Nord

Référence électronique

Adolfo Vera, "Inscrire les fantômes dans les villes », Appareil [En ligne], 22 | 2020, mis en ligne le 21 décembre 2020, consulté le 26 mars 2021. URL : http://journals.openedition.org/appareil/3488 ; DOI : https://doi.org/10.4000/appareil.3488

Ce document a été généré automatiquement le 26 mars 2021.

\section{(c) (i) (9)}

Appareil est mis à disposition selon les termes de la Licence Creative Commons Attribution - Pas d'Utilisation Commerciale - Pas de Modification 4.0 International. 


\title{
Inscrire les fantômes dans les villes
}

Innerver les corps pour résister

\author{
Adolfo Vera
}

\section{NOTE DE L'ÉDITEUR}

Texte écrit dans le contexte du projet de recherche Fondecyt Iniciación nº 11180754.

\section{Introduction}

1 Quand quelqu'un disparaît, on dit: «il n'y a plus de traces de lui ». La disparition, en fait, est le « résultat » de l'effacement de toute trace de quelqu'un. Or, qu'est-ce qu'une trace? Est-il possible d'effacer «toutes » les traces de quelqu'un, c'est-à-dire, tout résidu, tout reste d'une expérience ? Il est évident, en tout cas, que la stratégie de la disparition politique est de chercher à effacer, justement, toute trace qu'a laissée la vie de quelqu'un. C'est ainsi qu'on peut imposer la terreur. Ce que les stratégies de la terreur veulent faire disparaître avant tout, c'est le corps. Le corps en tant que trace. À l'inverse, la lutte des proches et des familles de disparus consiste à "faire apparaitre " (ou réapparaître) les traces de l'expérience du disparu. La trace acquiert, dans ce mouvement, un aspect éminemment politique : il s'agit de "démontrer " la "réalité » de l'existence de quelqu'un à partir de l'exhibition de l'accumulation de traces (documents divers: certificats, photos, œuvres; les signes, donc, d'une vie) qu'il a laissées. Cette accumulation de traces s'appelle «archive». Au Chili, par exemple, le travail du Vicariat de la solidarité, organisme dépendant de l'Église catholique qui se portait garant des droits de l'homme pendant la dictature d'Augusto Pinochet, consistait dans une grande mesure à établir des "dossiers" sur les disparus, comme autant d'archives qui contenaient des traces (des photos, des certificats de scolarité, des certificats de naissance, etc.). Ces dossiers permettaient, le cas échéant, de présenter des "preuves de vie» face à la justice et aux représentants de l'État terroriste qui niaient le passage de ces personnes dans leurs organismes (négation 
rendue possible, bien évidemment, par l'effacement de tout registre de ce passage). La trace a donc une importance juridique majeure. Elle est peut-être la seule manière de mettre en évidence le différend (au sens de Jean-François Lyotard), le tort radical, subi par les victimes de la disparition politique. D'autre part, les images aussi sont des traces et notamment les images techniques qui peuplent nos vies, depuis qu'au milieu du $\mathrm{XIX}^{\mathrm{e}}$ siècle est apparue la photographie. Quand ceux qui produisent les images travaillent leur statut de trace (et donc ses rapports avec l'inscription, la désinscription, la mémoire, l'oubli et avant tout la disparition), ils produisent des images politiques. C'est le cas d'artistes comme Christian Boltanski, Alfredo Jaar, Jochen Gerz, Marcelo Brodsky, Ernest Pignon-Ernest, etc., mais aussi de quelques expériences esthétiques collectives comme le Siluetazo argentin, qui a eu lieu en 1983 à Buenos Aires et d'autres villes du pays sud-américain. Cette expérience esthétique et politique était vouée à "montrer" au monde l'existence des disparus contre le négationnisme systématique des militaires et de l'extrême droite et pour ce faire, les possibilités d'« innervation » des corps par les images reproductibles techniquement, et donc leur rapport essentiel avec le geste, ont été fondamentales.

2 La trace (par exemple, l'empreinte que laissent les corps sur un support, comme pour la photographie) a-t-elle aussi une importance politique, au-delà de ses effets esthétiques? Si l'on comprend le terme esthétique à la manière de Jacques Rancière ${ }^{1}$, c'est-à-dire comme l'ouverture du sensible au commun, le partage de la sensibilité (ce qu'on peut, dans un moment historique, dire ou non, entendre ou non, voir ou ne pas voir), conséquence des dérèglements constants des rapports sociaux à l'intérieur de la communauté, partage où l'art a une fonction politique essentielle; si l'on entend donc les figurations que l'art peut faire surgir à l'intérieur de la communauté brisée par la violence, non pas comme des modalités de réunification ou de rédemption, mais de redistribution, on peut donc bien dire que ces figures-là sont autant de traces qui permettent l'apparition, par exemple, de la disparition en tant que " phénomène ", qui sera donc foncièrement spectral. C'est ce caractère spectral qui, comme nous le verrons, définit aussi l'essence de la trace, ce qui constitue les œuvres de photographes comme Gustavo Germano ou Marcelo Brodsky, mais aussi cette expérience collective que fut le Siluetazo argentin. L'art qui s'occupe de la disparition est un art spectral puisqu'il s'agit d'un art de la trace et de l'archive - un art de l'inscription, de l'écriture, de la mémoire et de l'oubli.

3 Le Siluetazo ${ }^{2}$ est l'une des pratiques esthétiques et politiques les plus connues parmi celles qui, en Amérique latine, ont essayé de faire acte de résistance face à la violence terroriste d'État. Ana Longoni ${ }^{3}$ a défini cette expérience - qui consiste à tracer les silhouettes des disparus à échelle humaine sur des supports urbains les plus divers et dans plusieurs villes d'Argentine en octobre 1983 - comme une conséquence de la volonté de représenter la " présence d'une absence ». Il s'agissait de l'initiative de trois artistes visuels (Rodolfo Aguerreberry, Julio Flores et Guillermo Kexel) qui, pour la réaliser, se sont associés avec les Madres de la Plaza de Mayo, les Abuelas de la Plaza de Mayo et d'autres organismes de militants et de défense des droits de l'homme. L'idée était inspirée par l'artiste polonais Jerzy SkĄpski, qui, à la fin des années 1970, avait créé une œuvre reproduite dans la revue Le courriel de L'Unesco, où l'on pouvait observer 24 rangées de petites silhouettes de femmes, d'hommes et d'enfants accompagnées du texte suivant : « Chaque jour à Auschwitz mouraient 2370 personnes, juste le nombre de figures ici reproduites. Le camp d'Auschwitz a fonctionné pendant 1688 jours, nombre 
imprimé sur cette affiche. On pense que le nombre de morts d'Auschwitz atteint les 4 millions ${ }^{4} »$.

\section{Le « paradigme spectral » des images techniques}

4 L'élaboration d'une philosophie esthétique et politique de la disparition est une tâche qui n'a pas été accomplie en Amérique Latine. Cette philosophie impliquerait d'établir un « paradigme spectral » pour penser les images en tant que surfaces d'inscription des traces errantes qui hantent, comme des fantômes, nos sociétés. Ce paradigme exige que le discours théorique qui essaiera d'en fournir les concepts nécessaires devra dépasser les limites, d'une part, des analyses qui posent le problème de la mémoire comme presque le seul à considérer (il s'agirait de considérer l'originaréité de l'oubli) et, d'autre part, d'une certaine conception des sociétés encore trop déterminée par les théories autour de l'hégémonie des discours (Antonio Gramsci, Louis Althusser, Michel Foucault), qui néglige de ce fait l'importance d'une philosophie de la technique pour penser les questions impliquées dans la disparition: le témoignage, l'archive, l'oubli, la spectralité. Il faut donc penser le paradoxe suivant: d'une part, prendre en charge la disparition implique, et pour l'esthétique et pour la pratique artistique, déstabiliser tous le fondement qui les soutiennent culturellement - le musée, les circuits établis (académiques et marchands) -, mais, d'autre part, on assiste à l'intérêt grandissant de ces institutions pour travailler sur la disparition et c'est avec elles que peuvent se récréer de nouvelles surfaces d'inscription pour accueillir ces pratiques. Cette tension n'empêche pas que l'on assume comme exigence analytique et critique le fait que les expériences esthétiques qui ont traité la question de la disparition dépassent le champ traditionnel de l'esthétique, non pas - comme c'était le cas pour les avant-gardes et les néo-avantgardes - pour élargir le territoire de l'art, mais tout simplement pour le dépasser en s'ouvrant - comme l'avait compris Lyotard avec sa relecture du concept de sublime chez Emmanuel Kant - à l'éthique, c'est-à-dire, au témoignage, puisque celui-ci est aussi un questionnement de la "vérité de l'art ».

Considérons, tout d'abord, par sa proximité avec le Siluetazo, “En memoria” («En mémoire ", 2000-2001) de Fernando Traverso. Il s'agit d'une série d'interventions urbaines à Rosario, en Argentine, autour des vélos utilisés par des membres de la résistance à la dictature, aujourd'hui disparus. Ces vélos, alors que leurs propriétaires ne revenaient pas les récupérer, restaient placés sur les murs, y projetant leur ombre des fois pendant des années. L'ombre fonctionne donc comme un index spectral, puisque c'est bien la disparition qui est inscrite sur le mur et non pas une présence pérenne. L'artiste a inscrit, grâce à une technique proche du graffiti, celle du pochoir, plusieurs ombres sur les murs de la ville - où jusqu'à aujourd'hui les gens préfèrent ce moyen de transport -, pour essayer de montrer cette présence spectrale, telle que les milliers d'ombres du Siluetazo. Ici, le « paradigme spectral » de la photographie est bien la clé de compréhension.

On a souvent évoqué la puissance symbolique de l'ombre, en ce qui concerne le sentiment du double et de l'inquiétante étrangeté. En outre, depuis le mythe de l'origine de la peinture raconté par Pline dans son Histoire Naturelle (mythe dit de la fille de Dibutade), les rapports entre trait, ombre, trace et représentation définissent la structure de l'œuvre de représentation visuelle. L'avènement de la photographie, puis du cinéma, n'ont fait que déplacer et replacer - dans le contexte de la reproduction 
technique - ces rapports. Dans ces appareils, l'ombre - ce qui, dans l'image, communique avec l'extériorité et l'«ailleurs"- joue un rôle toujours essentiel ${ }^{5}$. En pensant à la photographie, Philippe Lacoue-Labarthe a pu élaborer le concept de «double pelliculaire ${ }^{6}$ ». La photographie prend en charge, sur la nouvelle surface de reproduction (pelliculaire) qu'elle impose, tous les affects archaïques (crainte, séduction, obsession) propres aux rapports des sociétés avec les ombres et, comme le rappelle Otto Rank, les miroirs ${ }^{7}$.

7 Or, le geste de Traverso prétend assumer ces significations anthropologiques et les mettre en jeu face à l'exigence de la disparition. Il n'a pas de meilleur moyen pour cela que de faire appel aux virtualités de hantise qui sont, par définition, celles des mécanismes de reproduction comme le pochoir ou la photographie. Le but des silhouettes répétées par milliers sur les murs des villes argentines lors du Siluetazo était justement de peupler les consciences de ces fantômes que sont les disparus, qui peuvent apparaître n'importe où dans la ville, pour nous rappeler, grâce à leur présence qui n'est que le signe d'une absence insupportable, qu'ils (ne) sont (pas) là. Traverso, pour sa part, en utilisant la même méthode, a choisi un objet d'usage commun à Rosario, qui, de ce fait, se charge symboliquement (puisqu'il acquiert le statut d'un « double », d'un « revenant »); pour énoncer la possibilité que ces vélos que les disparus n'ont pas eu le droit de réutiliser pour aller au travail ou juste pour se promener, sont encore là et que, toujours rangés, ils attendent leurs anciens propriétaires (comme la société tout entière attend que justice soit faite).

Nous sommes face à une première détermination des rapports entre corps et images techniques. Celle-ci nous est donnée par la question du geste comme expression du corps et les objets techniques qui s'individuent avec lui. Selon Gilbert Simondon, on retrouve ici l'essence de l'esthétique comme rapport conflictuel entre les tendances magiques et techniques. Pour ce dernier, l'esthétique signifie la position du corps dans le contexte des «réticulations» que l'homme établit pour se repérer dans l'espace, d'une part "magiquement» (en établissant des "points clés » qui ont avant tout un caractère magique) et d'autre part «techniquement" (en faisant appel à des « schématismes » rationnels qui permettront la classification et la division à l'infini du réel), le geste technique apparaissant justement comme conséquence de ces deux manifestations essentielles de l'humanité, notamment comme un essai de les réorganiser unitairement; effort qui se trouvera, le plus souvent, être infructueux, du fait que le processus d'individuation qui les a séparés n'est pas réversible 8 . Cependant, l'esthétique permettra d'établir une conception du territoire comme un réseau de « points clés » interconnectés symboliquement.

9 Les vélos spectraux imprimés et reproduits par Traverso à Rosario sont bien, dans ce sens, des "points clés». Ils permettent, en tant qu'images techniques, un type d'individuation, qui est celui propre aux objets techniques selon Simondon. L'une des particularités les plus importantes de la philosophie de l'objet technique proposée par Simondon, consiste à penser celui-ci non pas (comme le font d'autres penseurs de la technique comme André Leroi-Gourhan, Jacques Derrida et Bernard Stiegler) comme une "prothèse "- extension et prolongation d'un organe du corps -, mais comme technicité qui crée des réseaux et des processus d'individuation. Le geste est un noyau fondamental d'un tel processus, puisqu'il est une sorte d'« action virtuelle », d'« acte en puissance» et l'on sait bien que, pour Simondon, il faut définir une «technoesthétique» qui ne se régira plus, comme pour Kant mais aussi pour l'art 
autonome défini par Theodor Adorno, par un jugement de goût ou une « contemplation désintéressée ", mais par une praxis technique en même temps qu'esthétique ${ }^{9}$. En effet, pour Simondon, un individu devient sujet quand il est à même d'assumer sa charge de pré-individualité (énergie biologique non liée) et de ce fait, se (dés)individualiser dans le collectif, en allant de l'émotion vers l'action et de l'affection à la perception. Toujours selon lui, "l'émotion, versant individualisé de l'action, résout le problème affectif parallèle au problème perceptif qui résout l'action ${ }^{10}$ ». De ce point de vue, l'occupation des villes par des images comme celles, spectrales, de l'intervention de Traverso, pourra permettre non seulement l'émotion face au contenu qu'elles transmettent (la violence extrême signifiée par la pratique totalitaire de la disparition politique), mais aussi le passage à l'action, donc à la politique. Quoi qu'il en soit, il est vrai d'ailleurs que ce processus peut aussi être celui de la publicité (un artiste comme Alfredo Jaar a travaillé cette question dans ce sens), dont l'émotion des images pourrait se résoudre dans l'action d'acheter. C'est un sujet sur lequel Simondon lui-même avait réfléchi, dans un texte sur la publicité ${ }^{11}$. Pour lui, l'effet de halo est même la conséquence d'une attitude psychique, qui consiste à se concentrer dans l'aspect purement esthétique, en le séparant $\mathrm{du}$ moment technique, d'un objet. La publicité opère en faisant artificiellement et idéologiquement cette séparation, avec la finalité d'inciter à la consommation de marchandises. De cette façon, les images techniques reproduites par Traverso en Argentine permettront, en activant le geste et l'action, que le rapport entre le moment technique et le moment esthétique produisent une appropriation collective des émotions concernées, c'est-à-dire, la spectralité.

Revenons au Siluetazo de 1983. Une fois l'idée mise au point, elle est présentée et approuvée par les Mères. Cependant, selon la reconstitution réalisée par Longoni, on peut signaler quelques points qui indiquent la «sagesse " politique, philosophique et même esthétique des Mères, par rapport à la question de la disparition. Tout d'abord, elles s'opposent à l'idée de "singulariser» les silhouettes avec des caractéristiques propres aux "portraits» des disparus. Pour elles, il faut trouver une autre solution pour indiquer le caractère «transpersonnel » de la disparition, au-delà de tout crime déterminé dans le cadre du droit pénal (on tue toujours quelqu'un de spécifique, avec un nom et un visage, mais quand on fait disparaître quelqu'un, il n'y a plus de visage et son nom est le nom d'un fantôme). D'autre part, une discussion a eu lieu entre les Mères et certains artistes autour de l'idée - défendue jusqu'à aujourd'hui par les Mères et qui a impliqué que certains les appellent «les folles de la place de Mai»- de «la réapparition en vie " des disparus. Cette idée supposait, pour les Mères, qu'on ne pouvait pas représenter les silhouettes déployées sur le sol puisque cela signifierait qu'on les identifie immédiatement à des cadavres, ce qui était - toujours selon Longoni - l'idée de certains artistes voulant en profiter pour évoquer la technique policière des dessins de silhouettes de cadavres, mais aussi la responsabilité des militaires représentés directement comme des «assassins» (ces artistes, ne comprenant pas, pourrait-on dire, que le crime de la disparition est encore pire que celui de l'assassinat).

11 D'un point de vue théorique, il faut signaler que les effets esthétiques et politiques du Siluetazo correspondent à ce que l'on peut appeler les "effets sociaux" de la reproduction technique de l'image (même s'il s'agit ici d'une technique quelque peu artisanale, comme l'est celle du pochoir). Ces effets, on le sait bien, ont été décrits par Walter Benjamin ${ }^{12}$. Or, selon ce dernier, cette importance se situait juste au milieu d'une crise : la fin du règne de la «valeur cultuelle » de l'image et le début de celui 
propre à sa "valeur d'exposition ». Cette crise, d'après lui, impliquait que si depuis ses origines les plus archaïques, l'image avait une fonction que l'on peut définir par le terme de "magie », c'est-à-dire, en manifestant, dans le culte, la présence réelle des êtres invisibles qui contrôlent le devenir, l'approche sécularisée déjà installée à la Renaissance - approche qu'il faut comprendre comme intimement liée à ce que Benjamin appelle «valeur d'exposition » - va imposer un rapport qu'il définira comme " politique » avec l'avènement de la reproduction mécanisée. Ce rapport est déterminé par les conditions de production, qui permettent un développement technologique particulier : celui que rend possible la reproduction technique. Cependant, il s'agit bien là d'une crise, donc d'une tension et d'un conflit dont la déchirure apparaît dans l'image moderne. Ce conflit se manifeste avec une puissance particulière, selon Benjamin, dans les portraits photographiques, puisque si, d'une part, toute photographie, en tant que produit de la reproduction technique, implique l'exacerbation de la valeur d'exhibition contre la valeur cultuelle, d'autre part, le portrait y signale, en tant que représentation du visage humain, "l'ultime retranchement de la valeur cultuelle », c'est-à-dire de l'aura. Selon Benjamin, « le culte du souvenir des êtres aimés, absents ou défunts, offre au sens rituel de l'œuvre d'art un dernier refuge. Dans l'expression fugitive d'un visage humain, sur d'anciennes photographies, l'aura semble jeter un dernier éclat. C'est ce qui fait leur incomparable beauté, toute chargée de mélancolie ${ }^{13}$ ». En revanche, avec les photographies prises par Atget à Paris autour de 1900, on pourra vérifier une émancipation presque totale du moment politique des images, du fait de la disparition du visage humain dans ces photos et donc de l'autonomie de l'image par rapport au « culte du souvenir des êtres aimés ». Étant vidés de toute humanité, Benjamin les définissait comme des photos de " scène du crime ", où tout nous oblige à chercher les indices, les traces et les motifs de cette, disons, inhumanité criminelle. C'est le même phénomène, comme on vient de le voir, qui s'est produit lors de l'action appelé Siluetazo.

Le rapport, donc, du Siluetazo, en tant qu'œuvre collective qui naquît de l'alliance d'artistes et de militants politiques autour de la « réalité spectrale » de la disparition et de l'image produite par la reproduction technique, est bien un rapport politique, au sens de Benjamin. L'idée des Mères de ne pas reproduire les visages des silhouettes est frappante dans sa proximité de la définition du moment politique de l'image selon ce dernier. Les artistes, néanmoins, se situant plus du côté de la définition «magique » de l'image, considéraient que la représentation du visage rendrait plus « humaines » ces silhouettes. On le sait aussi grâce à Benjamin, l'enjeu fondamental de la pensée politique après la catastrophe, est bien celui de l'inhumanité de l'humain. C'est peutêtre pour cela qu'il faudra, dans l'élaboration d'une philosophie politique de la disparition, qui doit considérer les discours sur les images techniques comme un moment essentiel, distinguer les notions de «trace » et d'« empreinte »: la première nous conduisant plutôt du côté de ce qui, en n'étant plus là, permet que son inscription demeure encore sous le signe de la spectralité; la seconde du côté d'une identité à soi d'un objet inscrit comme inamovible. La trace donc ouvrant vers une pensée de la politique contemporaine (l'inhumanité du crime comme partie prégnante de notre quotidienneté), l'empreinte vers une pensée de la religion de l'image (le voile de Véronique).

13 Posons-nous alors la question suivante : peut-on définir cette expérience esthétique collective comme un "effet de fantôme "? On peut envisager le Siluetazo, grâce aux possibilités données par les techniques de reproduction d'images (d'abord le pochoir 
pour la réalisation, mais postérieurement la photographie pour la diffusion mondiale de l'événement), comme une manière d'accueillir socialement, en les métaphorisant, le trauma et la catastrophe : une autre façon de définir l'accueil des fantômes. Un « effet de fantôme " produit par une expérience esthétique (soit celle d'une œuvre individuelle ou celle d'une expérience collective) pourra donc être défini comme la capacité, par des moyens techniques spécifiques - notamment ceux déterminés par la reproduction d'accueillir les doubles de la société, ses fantômes, les oubliés, ceux qui ne sont pas là, puisqu'ils n'ont pas de langue ni de voix : les vaincus de l'histoire (Benjamin) dont les disparus constituent un cas extrême. Ces expériences devront se moduler sur la logique de la revenance, donc de la mémoire et de l'histoire, et non pas (comme une grande partie de l'art contemporain) sur celle de la pure image considérée comme une réalité en elle-même.

\section{La trace. Corps, Innervation, Machine}

Dans Freud et la scène de l'écriture, Derrida se propose d'étudier « les contradictions systématiques dans l'exclusion onto-théologique de la trace ${ }^{14} \ldots$ », mais aussi «le refoulement de l'écriture comme de ce qui menace la présence et la maitrise de l'absence ${ }^{15} »$. On est donc sur le même terrain philosophique que De la grammatologie et La voix et le phénomène : la déconstruction de la métaphysique de la présence. Il s'agirait donc de déconstruire ce que la «répression logocentrique» a dénié "comme métaphore didactique et technique, comme matière servile ou excrément ", c'est-à-dire ce qui, en Occident, a nié «le corps de la trace écrite ${ }^{16} »$. Ce corps se constitue à l'intérieur d'une certaine machine d'écriture, qui appartient à la structure d'un appareil particulier: l'appareil psychique. Il faudra donc parler d'une «machine d'écriture psychique», qui consiste à inscrire des traces (mnésiques) qui seront travaillées par l'appareil psychique. Dans les Notes sur le bloc-notes magique, Sigmund Freud décrit le fonctionnement de cet appareil à partir d'une métaphore : le petit engin qui permet d'écrire sur une feuille de papier et d'effacer ce qui a été écrit sans stylo ni effaceur, mais qui dans une couche de cire conserve quand même les traces de l'écriture, uniquement observables sous une lumière particulière. Or, pour Derrida, il ne s'agit pas tant d'observer dans le texte freudien la qualité ou la précision de la métaphore, mais de se demander «quel appareil il faut créer pour représenter l'écriture psychique et ce que signifie, quant à l'appareil et quant au psychisme, l'imitation projetée et libérée dans une machine, de quelque chose comme l'écriture psychique $^{17}$ ».

15 Selon Derrida, on assiste chez Freud au passage d'une conception où le système d'inscription de traces fonctionne sur un modèle «naturel " (le frayage des neurones comme ce qui définit le fonctionnement de la mémoire), l'écriture en étant absente, à une conception selon laquelle la structuration de la trace doit être représentée comme écriture. Selon Derrida, le problème de la première explication, qui se veut digne de la méthode des «sciences naturelles», est qu'elle «rend l'appareil presque inimaginable». Derrida continue : «La croix d'une telle explication [...] c'est qu'il lui faut rendre compte à la fois, comme le fera la Note, trente ans plus tard, de la permanence de la trace et de la virginité de la substance d'accueil, de la gravure des sillons et de la nudité toujours intacte de la surface réceptive ou perceptive : ici des neurones ${ }^{18}$ ». Il est question de "gravures des sillons », d'inscription et de permanence 
des traces, mais surtout de «frayages ». Selon Freud, expliqué toujours par Derrida, il y aurait deux sortes de neurones, ceux qui retiennent l'information du fait du frayage des impressions provenant du monde extérieur (les neurones de la mémoire) et ceux qui n'en retiennent pas (les neurones de la perception). Il s'agirait donc de la « résistance et par là même [de l'] ouverture à l'effraction de la trace ${ }^{19}$ ». La trace va être décrite par Derrida, à partir de Freud, comme la "différence invisible et insaisissable entre les frayages ${ }^{20} »$. Selon le premier, il n'y a néanmoins pas lieu de considérer ces différences en termes de qualité ou de quantité, comme le ferait par exemple la physique ou la biologie et comme essayait de le faire Freud lui-même dans l'Esquisse d'une psychologie scientifique ${ }^{21}$ de 1895 . C'est en raison de cela qu'une analyse de la mémoire doit nécessairement échapper aux méthodes de la phénoménologie et des sciences naturelles.

On se trouve donc face à un système où il n'y a que des inscriptions. Derrida montre dans son analyse comment la pensée de Freud évolue d'une considération neurologique à une autre (à propos de laquelle le petit texte sur le Bloc-notes a une place d'honneur) où l'écriture est le motif central. Il s'agit donc de différents types d'inscriptions ${ }^{22}$. On est face, selon Derrida, à une « lithographie d'avant les mots ${ }^{23}$ ». Ce qui se constitue de cette façon est donc une « écriture psychique ». Cette écriture " ne se laisse lire à partir d'aucun code ${ }^{24} »$. Cela veut dire, entre autres choses, qu'il n'y a pas lieu d'opposer un signifiant à un signifié : lorsque l'on est sur le terrain de l'appareil psychique, on est audelà des oppositions qui configurent la métaphysique de la présence. "Qu'on ne distingue [écrit Derrida] le signifié du signifiant, avec Saussure, que comme les deux faces d'une même feuille, cela n'y change rien. L'écriture originaire, s'il en est une, doit produire l'espace et le corps de la feuille elle-même ${ }^{25}$ ". Si l'on veut être fidèle à Freud, on ne doit pas, selon Derrida, attribuer à cette écriture une quelconque fonction de «traduction ». Cela impliquerait penser qu'il y a déjà un texte intact, présent à luimême comme une présence à transcrire. Cela veut aussi dire que l'ensemble de textes, tissés de traces mnésiques, qui conforme l'appareil psychique, se configurent comme des archives « qui sont toujours déjà des transcriptions ${ }^{26}$ ». Ce sera le « mal d'archive ». Derrida : «Tout commence par la reproduction. Toujours déjà, c'est-à-dire dépôt d'un sens qui n'a jamais été présent, dont le présent signifié est toujours reconstitué à retardement, nachträglich, après-coup, supplémentairement : nachträglich veut dire aussi supplémentaire ${ }^{27} »$. On retrouve ici un concept clé pour la déconstruction de la négation de l'écriture par la tradition métaphysique. En fait, toute la deuxième partie de l'ouvrage De la grammatologie porte sur le mépris, la négation et, pourrait-on presque dire, la peur qu'un philosophe comme Jean-Jacques Rousseau a pu ressentir face à l'écriture. Derrida montrera aussi comment ce mépris affecte des penseurs contemporains comme Claude Lévi-Strauss. Or, la notion source qui articule cette véritable guerre contre l'écriture - dans les recherches de Lévi-Strauss, on pouvait vérifier comment l'introduction de l'écriture était la cause de la "décadence " de certains peuples primitifs - est celle de "supplément " : l'écriture est conçue comme cet artifice qui essaie de remplacer la liberté et la pureté de la parole, en imposant les codes et les normes de la tradition, en séparant les sujets de la nature. L'écriture est en ce sens la manifestation la plus originaire de la technique. Elle est avant tout une prothèse. L'on pourrait dire que si la technique ne peut pas être pensée véritablement par la métaphysique de la présence, qui depuis Platon jusqu'à Heidegger la rejette comme " anti-naturelle ", c'est bien parce que la technique, en tant que supplément, dépasse toute présence et "apparaît " comme "retardement», conformément à la 
logique temporelle de l'après-coup. C'est donc la répétition (qui communique nécessairement avec la puissance de mort) qui produit le sens, comme un effet de machine. La trace est le lieu de cette répétition, contre toute idée d'unicité et d'origine (on sait aussi comment Benjamin pense la trace par contradiction avec l'aura). La trace inscrit : elle se fraye un chemin, le rompt, et de cette façon elle constitue la spatialité de l'espace. Or ce "travail de taupe », comme l'appelle Derrida ${ }^{28}$, ouvre le devenir-espace du temps, sa discontinuité radicale, sa non-coïncidence avec soi-même, ce qu'on a appelé l'anachronisme comme fonction de la spectralité. La trace inscrit et ouvre, c'està-dire désinscrit en même temps, et c'est ainsi que fonctionne l'inscription spectrale : l'inscription existe, reste, marque, imprime, mais elle appartient au mouvement de la revenance, donc elle peut (elle doit) se désinscrire à chaque fois. La trace est donc une très particulière «impression ». "Celle-ci [écrit Derrida] a laissé une trace travailleuse qui n'a jamais été perçue, vécue dans son sens au présent, c'est-à-dire en conscience. Le post-scriptum qui constitue le présent passé comme tel ne se contente pas, comme l'ont peut-être pensé Platon, Hegel et Proust, de le réveiller ou de le révéler dans la vérité. Il le produit ${ }^{29}$ ".

17 La trace est donc, dans le sens de sa détermination comme revenance, vouée à l'effacement. Toute trace, par définition, est susceptible d'être effacée. Les frayages des traces sur les surfaces d'inscription de l'appareil psychique sont nécessairement reçus par la conscience à retardement (nachträglich, après-coup) et cela implique qu'il y a, à la fois, inscription et effacement. "Les traces [écrit Derrida] ne produisent donc l'espace de leur inscription qu'en se donnant la période de leur effacement ${ }^{30} »$. C'est tout-à-fait le cas des actions "éphémères » comme celles de Traverso ou celles d'Ernest PignonErnest (sur lesquelles nous reviendrons) et, bien sûr, du Siluetazo argentin. Un art " pérenne » n'est pas aussi à même de « représenter » la spectralité.

On pourrait donc dire que la trace est un type assez particulier de marque. En tant que telle, elle laisse une «impression » et implique alors nécessairement la manifestation de la spectralité. Pour comprendre le fonctionnement d'une marque ayant le caractère de trace, il nous faut mettre en rapport la mémoire, la technique et la spectralité. Cela signifie introduire la question de l'archive.

19 La spectralité fonderait donc bien la structure générale de l'archive ${ }^{31}$. En outre, d'un point de vue temporel, c'est-à-dire celui qui est central quand il s'agit de penser ce qu'on appelle la "mémoire historique ", la spectralité impose à la considération philosophique la question de la «survivance». Concernant l'histoire de l'art, on connaît les analyses qu'en a faites Georges Didi-Huberman dans son livre L'image survivante $^{32}$. Pour l'instant, concentrons-nous sur les conclusions que Derrida y relève. Pour ce dernier, « la survivance ne signifie plus la mort et le retour du spectre, mais le survivre d'un excès de vie qui résiste à l'anéantissement ${ }^{33} »$. Or, où, dans quel lieu, dans quelle configuration matérielle trouve-t-on la demeure de cet excès de vie ? Dans les murs d'une ville, par exemple (les murs où Pignon-Ernest imprime ses images reproduites mécaniquement) ? L'archive, peut-elle être considérée comme une telle demeure ? En tout cas, si on peut l'y trouver, on ne la trouvera pas « au passé ». C'est l'« avenir » qui compte, mais l'avenir n'est jamais la projection du passé ou du présent, comme celui-ci n'est pas non plus l'actualisation du passé. L'avenir est la voix du spectre qui vient dire : « The time is out of joint ${ }^{34}$ ». Puisque, comme le signale Derrida, même s'il ne répond pas, "cela parle, un fantôme $\mathrm{e}^{35}$ ». Cela parle, « un peu comme ce répondeur automatique (answering machine) dont la voix survit à son moment 
d'enregistrement ${ }^{36}[. .]$.$» . La technique y est donc nécessairement mêlée. Si elle y est$ mêlée, la répétition et la pulsion de mort aussi ; mais, selon Derrida, c'est la vie comme avenir qui triomphe. Toutefois, cela ne veut pas dire que la violence en est exclue, au contraire : «Dans tous les cas, il n'y aurait pas d'avenir sans répétition. Et donc, peutêtre, dirait Freud (ce serait alors sa thèse), pas d'avenir sans le spectre de la violence œdipienne qui inscrit la sur-répression dans l'institution archontique de l'archive, dans la position, l'auto-position ou l'hétéro-position de l'Un et de l'Unique, dans l'arkhé nomologique. Et la pulsion de mort ${ }^{37}$ ». C'est tout à fait le "mal d'archive » dont parle Derrida : il n'y aurait pas d'archive sans la destruction anarchivante qui produit « cela même qu'elle réduit, parfois en cendres, et au-delà ${ }^{38}$ ».

\section{Trace, dessin, ombre}

Pour Derrida, le mythe de l'origine de la peinture raconté par Pline dans le Livre XXXV de l'Histoire naturelle est en ce sens révélateur. En fait, ce qu'aurait inventé Dibutade à la demande de sa fille, qui, à l'instant où son amoureux est parti, a inscrit sur le mur le reflet de son ombre et a demandé à son père de l'y imprimer - comme les proches des disparus argentins impriment leurs ombres sur les murs des villes-, ce serait une " écriture de l'ombre », inaugurant de ce fait " un art de l'aveuglement ${ }^{39}$ ». « Détachée du présent de la perception, tombée de la chose même qui se partage ainsi, une ombre est une mémoire simultanée, la baguette de Dibutade est un bâton d'aveugle ${ }^{40} »$. En commentant Maurice Merleau-Ponty, Jacques Derrida va signaler que le visible est produit par l'invisible, plus précisément « hanté » par lui : l'invisibilité est la hantise de la visibilité. Le "point d'aveuglement » signalerait le moment précis de l'union entre visibilité et invisibilité. Le visible "produirait ainsi de l'aveuglement, par émanation ${ }^{41} \ldots$ ». Or, ce qui nous intéresse ici, est le fait que ce processus se produit comme conséquence de la structure même de la trace. En fait, elle serait constituée par ce que Derrida définit comme «le retrait ou l'éclipse, l'inapparence différentielle du trait ${ }^{42}$ ». Derrida de se demander : "Que penser maintenant du trait une fois tracé ? Non pas de son frayage et du trajet inaugural de la trace, mais de ce qui en reste ${ }^{43}$ ? ». « Un tracé ne se voit pas ", répond Derrida, puisqu'il n'est au fond qu'une limite, un seuil.

[La] ligne même : qui donc n'est plus ce qu'elle est, car elle ne se rapporte dès lors jamais à elle-même sans se diviser aussitôt, la divisibilité du trait interrompant ici toute identification pure, et formant, on l'aura sans doute compris maintenant, notre hypothèque générale pour toute pensée du dessin, à la limite inaccessible en droit [...]. Rien n'appartient au trait, donc au dessin et à la pensée du dessin, pas même sa propre "trace"44.

21 Il faut également bien avoir à l'esprit que si la trace n'appartient même pas au trait qui l'a tracé, qui la configure et la fait "apparaître", c'est bien parce que, de par sa structure foncièrement spectrale, elle se désinscrit au même instant qu'elle s'inscrit, disparaît alors et fait droit à l'invisibilité qui la constitue. Dans ce sens, la trace fonctionne comme une ombre : elle divise ce qui apparaît, renvoyant les choses à son double, à son fantôme: comme les fantômes qui peuplaient les villes argentines en 1983.

Du reste, en ce qui concerne les rapports entre trace, inscription et appareils, nous ne pouvons pas négliger les analyses qu'a fait Jean-Louis Déotte à ce sujet ${ }^{45}$. Si l'on part de l'hypothèse que les appareils sont ces « inventions techniques » (au sens simondonien) qui vont «faire époque » du fait qu'elles ont la capacité de changer les modalités de la 
perception humaine, on doit penser que toute trace, pour qu'elle existe, pour qu'elle " apparaisse », doit être appareillée ${ }^{46}$. Cela veut dire, également, qu'il y a des époques où les traces sont appareillées de diverses façons. Par exemple, à l'époque de l'appareil perspectif, les traces apparaissent dans l'espace, ce qui n'existait pas à l'époque médiévale, où primait la logique de l'incarnation - l'appropriation de la chair par l'esprit lors du rituel de la communion - et non pas par celle de la représentation (qui ne se constitue qu'à partir des Traités de perspective inaugurés par Filippo Brunelleschi au $\mathrm{XV}^{\mathrm{e}}$ siècle). Elles sont donc déterminées par le rapport entre le point de sujet et la ligne d'horizon. Cependant, pour Déotte, c'est l'horizon la condition de possibilité du sujet. Si celui-ci peut avoir des "projets ", c'est bien parce qu'il appartient à l'époque projective déterminée par l'appareil perspectif ${ }^{47}$. Selon lui: «L'expérience du sujet moderne tiendra donc à être exclusivement horizontale, rabattant la transcendance du divin sur l'immanence de ce qui se projette comme traces pour un sujet sur un écran transparent ${ }^{48} »$.

\section{Corps, trace, appareil : l'innervation}

Cette immanence est aussi celle des villes. Une ville - c'est une hypothèse - peut être considérée comme une archive. Pour être plus précis : la ville fonctionne aussi - comme tout ce qui existe - selon la logique, spectrale, de l'inscription/désinscription des traces, étudiée par Derrida en ce qui concerne l'appareil psychique, mais que nous pourrions - à la suite des travaux de Jean-Louis Déotte ${ }^{49}$ - étendre à l'appareillage des villes. Cet appareillage - par exemple, celui de la perspective ou du cinéma - définit des modèles de représentation, mais avant tout des types d'existence (de circulation, par exemple, en ce qui concerne un autre appareil important du XIX ${ }^{e}$ siècle : les Passages). Un type de représentation essentiel pour l'époque de la perspective sera donc celui de la projection (tout est projeté, la vie la plus quotidienne qui implique qu'on a des " projets » pour l'avenir, mais aussi les projets politiques globaux en ce qui concerne les utopies par exemple); pour l'époque de la photographie, du cinéma et des Passages, ce sera la fantasmagorie ${ }^{50}$.

Dans le contexte de cet appareillage des villes, la représentation qui en est déterminée ne dépend pas uniquement de l'appareil psychique, mais aussi - et peut-être avant tout - du corps. C'est ici qu'apparaît chez Benjamin le concept essentiel et fort complexe d' "innervation ». Benjamin le reprend de la physiologie du XIX siècle, qui définissait les processus de transmissions des pulsions électriques par les voies nerveuses. Or, dans le texte sur L'œuvre d'art, Benjamin reprend le concept à partir de l'utilisation qu'en avait fait le théoricien de l'art Konrad Fiedler ${ }^{51}$, pour qui la vue du peintre innerve sa main en lui octroyant les moyens matériels et techniques propres à la peinture (pas de "vue » sans être passé au préalable par le toucher et le contact avec la matière, c'est donc la main du peintre qui voit). Benjamin a retravaillé ce concept dès les années 1920, dans son texte sur un «Programme pour un théâtre d'enfants prolétarien ", écrit sur commande de la révolutionnaire russe Asja Lācis qui lui avait demandé une théorisation de son activité théâtrale avec des enfants orphelins dans le Moscou des années $1920^{52}$. Comme l'explique Bruno Tackels :

Dans ce programme de circonstance, qui se révèle être, soixante ans plus tard, la matrice d'une théorie du théâtre, l'enjeu est de démonter la dictature de l'idéologie, cet univers des phrases qui ne s'adressent qu'à ceux qui les connaissent déjà [...]. Cette récusation de l'idéologie se laisse traduire sur le plan esthétique par la 
condamnation des dangereuses magies de la pure imagination. L'homme d'imagination est un être enfermé. Cette faculté dominante, qui est pure réception, pure passivité de l'homme anesthésié par la première technique (ici la peinture, comme primat du regard extatique) doit donc être transformée: innervée.

L'imagination doit être traversée par le travail tactile du geste ${ }^{53}$ [...].

C'est un geste qui est capable d'imaginer, donc. Pour sa part, dans un livre récent, Peter Szendy ${ }^{54}$ a expliqué comment nos rapports aux villes, en ce qui concerne nos gestes et nos actions, passent nécessairement par l'appareillage des corps produit par les images techniques reproductibles, notamment celles du cinéma ${ }^{55}$. Du fait de l'innervation, les images que l'on perçoit se transforment en actions. D'un point de vue technoesthétique qui, ici, coïncide pleinement avec celui de Benjamin (et que, il faut le dire, n'est pas celui de Szendy, qui reste attaché à un marxisme trop rigide ${ }^{56}$ ) ces actions ne sont pas, par définition, réductibles à l'" effet de halo » ou au « fétichisme de la marchandise ", mais elles peuvent (même s'il s'agit d'une idée pour l'avenir, d'un projet justement, peut-être d'une utopie) faire réagir aux sujets dans le sens de l'émancipation et de la critique. Pour le dire dans une phrase: il y a bien une innervation publicitaire et marchande, qui domine nos vies, mais il y a aussi (ne seraitce que comme possibilité) une innervation esthétique et politique vouée à l'émancipation.

Un aspect essentiel de cette possibilité émancipatrice et critique que Benjamin établit à partir de l'innervation des appareils, est celle du moment collectif du processus. Le moment politique d'un art non auratique comme l'est le cinéma ${ }^{57}$ est celui du collectif (un collectif qui se libère de sa condition de masse, comme le peuple deleuzien, du fait qu'il n'est pas là, qu'il manque, qu'il faut l'inventer à chaque fois). Comme l'explique Bruno Tackels: "Cet art politique est une manière, non pas de retourner au jeu originaire et antérieur à toute œuvre auratique, mais plutôt de faire voir que l'aura naît avec le jeu et que le collectif ne se constitue que sur fond du jeu - comme faire-œuvre, comme l'œuvre en train de se faire ${ }^{58} »$.

C'est justement dans ce sens que l'on peut interpréter le Siluetazo comme un art politique puisque collectif, du fait qu'il jouera aussi avec l'énorme puissance évocatrice de l'ombre (puissance du double, de la répétition, de la spectralité), mais avant tout parce qu'il sera un travail collectif où les images techniques auront pu innerver les corps pour les libérer du poids de l'absence et de la disparition en leur permettant, donc, dans un geste qui est aussi une action politique, de représenter cette absence et cette disparition.

\section{Conclusion}

L'appareillage des corps qui pourrait les émanciper - comme disait Benjamin dans son programme pour un théâtre prolétarien - en les libérant de l'enfermement de l'imagination extatique et isolée et l'innervation produite par des appareils de production d'images techniques reproductibles, qui permettront la transformation de l'émotion en action, d'images en gestes, pourraient être considérés comme les noyaux théoriques pour une pensée autour des résistances esthétiques face à la violence extrême totalitaire. Ces deux phénomènes - appareillage des corps et transformation de l'imagination en action et praxis politique - ont été, bien évidemment, au cœur des pratiques et des analyses artistiques du $\mathrm{xx}^{\mathrm{e}}$ siècle, notamment en ce qui concerne, pour 
ne citer qu'un texte devenu classique, les rapports entre art, action et participation ${ }^{59}$. Or, qu'est-ce qui arrive quand ce type de problématiques (qui appartiennent, depuis longtemps, aux institutions les plus normatives qui soient, comme l'université et les musées) doivent faire face à une violence telle qu'elle est capable de détruire la communauté humaine, comme c'est bien le cas de la stratégie de la disparition politique utilisée par les régimes totalitaires pour imposer la terreur? La question de la résistance acquiert ici un tout autre sens et il n'est pas autre que le sens de la survie. Il faut entendre ici survie comme survivance et celle-ci comme survivance des fantômes. Comme l'ont montré les psychanalystes Nicolas Abraham et Maria Torok ${ }^{60}$ - dont les théories ont énormément influencé Derrida - les fantômes (toujours en provenance d'un autre) s'encryptent dans le moi, c'est-à-dire s'inscrivent dans un endroit qu'on ne peut pas identifier, une sorte de double du moi, que l'on ne peut pas contrôler. Cette inscription fantomatique produira des véritables émanations de hantise, c'est-à-dire, des affects non liés qui sont à la quête d'un endroit où s'établir. On pourrait étendre cette théorie à la société tout entière. Comme l'a aussi montré Derrida, l'hospitalité n'est autre chose que l'exercice de cet accueil, mais il exige le travail de création de surfaces d'inscription. On assume souvent que, en ce qui concerne l'art, l'espace privilégié de cet accueil est celui des institutions, notamment les musées. Il est évident qu'ils ont, dans ce sens, accompli une fonction politique très importante. Les villes aussi peuvent être des lieux d'accueil, surtout pour des expériences collectives de résistance. Ça a été le cas du Siluetazo argentin.

29 Ça a aussi été le cas de quelques artistes, dont peut-être le plus important, en ce qui concerne les occupations de villes par des images techniques qui représentent les hantises contemporaines, est celui d'Ernest Pignon-Ernest. On pourrait mentionner deux de ses interventions: la première, en 1971, à Paris, pour commémorer le centenaire de la Commune, avec des sérigraphies étendues comme des corps gisants sur des endroits où le sang des communards a coulé. Autour de cette intervention, PignonErnest a dit: «[...] il ne m'était pas possible de rendre compte d'un tel événement au moyen d'un tableau qui irait prendre place dans une exposition. Cela me paraissait un non-sens: la négation même de l'esprit de la Commune ${ }^{61}$ ». Il ajoute: «Il fallait témoigner au ras de sol, réinvestir les lieux chargés d'Histoire ${ }^{62}[\ldots]$ ». Il s'agirait donc d'un témoignage au ras du sol, ou dans les murs : c'était le cas de la seconde action qu'on voudrait mentionner ici. En 1981, comme geste de solidarité avec les artistes chiliens qui souffraient comme le reste de la population les effets de la violence d'État, Ernest Pignon-Ernest décide d'aller à Santiago pour faire un hommage à Pablo Neruda, le poète mort quelque jours après le coup d'état de Pinochet et dont sa mort (même s'il était atteint d'un cancer très avancé) a probablement été la conséquence d'un assassinat planifié par les militaires ${ }^{63}$. La sérigraphie réalisée par Pignon-Ernest laissait un espace blanc (le célèbre " poncho » du poète) pour permettre que les artistes et les résistants, qui allaient coller les sérigraphies dans différents endroits de la capitale du Chili, introduisent des phrases, des mots, des signes, en permettant une sorte d'individuation psychosociale et technoesthétique, pour le dire comme Simondon. Elle sera aussi, comme ce fut le cas pour l'intervention de Traverso et pour le Siluetazo, une innervation des corps qui leur aura permis d'établir des surfaces d'inscription pour accueillir les fantômes. 


\section{BIBLIOGRAPHIE}

Baltrušaitis Jurgis, Le miroir : essai sur une légende scientifique. Révélations, science-fiction et fallacies, Paris, Le Seuil, 1978.

Benjamin Walter, «L'œuvre d'art à l'époque de sa reproduction mécanisée », in Écrits français [1933-1940], Paris, Gallimard, coll. « Folio essais », 2006, p. 149-248.

Benjamin Walter, Paris, capitale du XIX siècle. Le livre des Passages [1939], trad. de l'allemand J. Lacoste, Paris, Le Cerf, coll. « Passages », 1989.

Déotte Jean-Louis, L'époque de l'appareil perspectif. Brunelleschi, Machiavel, Descartes, Paris, L'Harmattan, coll. « Esthétiques », 2001.

Déotte Jean-Louis, L'époque des appareils, Paris, Lignes/Manifeste, 2004.

Déotte Jean-Louis, « La ligne d'horizon », Ici et ailleurs, 8 juin 2011. URL : http://ici-et-ailleurs.org/ spip.php?article155 [consulté le 15/9/2020]

Déotte Jean-Louis, Qu'est-ce qu'un appareil ? Benjamin, Lyotard, Rancière, Paris, L'Harmattan, coll. « Esthétiques », 2007.

Déotte Jean-Louis, Walter Benjamin et la forme plastique. Architecture, technique, lieux, Paris, L'Harmattan, coll. « Esthétiques », 2012.

Derrida Jacques, « Freud et la scène de l'écriture », in L'écriture et la différence, Paris, Seuil, coll « Point Essais », 2014.

Derrida Jacques, Mal d'archive. Une impression freudienne, Paris, Galilée, coll. « Incises », 1995.

Derrida Jacques, Mémoires d'aveugle. L'autoportrait et d'autres ruines, Paris, Réunion des Musées Nationaux, coll. « Parti pris », 1990.

Didi-Huberman Georges, L'image survivante. Histoire de l'art et temps des fantômes selon Aby Warburg, Paris, Minuit,coll. « Paradoxe », 2002.

Fiedler Konrad, Essais sur l'art, trad. de l'allemand D. Wieczorek, Besançon, Les éditions de l'imprimeur, 2002.

Fiedler Konrad, Sur l'origine de l'activité artistique, trad. de l'allemand I. Parvu et I. Rotermund, Paris, Rue d'Ulm, 2008.

Freud Siegmund, « Esquisse d'une psychologie scientifique », in La naissance de la psychanalyse, $7^{\mathrm{e}}$ édition, trad. de l'allemand A. Berman, Paris, Puf, 1996.

Lācis Asja, Walter Benjamin et le théâtre pour enfants prolétarien, trad. de l'allemand P. Ivernel, Paris, Le Portique, coll. « Carnets », 2007.

Lacoue-Labarthe Philippe, « Portrait de l'artiste, en général », in Écrits sur l'art, Genève, Les presses du réel, 2009, coll. « Mamco », p. 39-79.

Longoni Ana et Bruzzone Gustavo (dir.), El siluetazo, Buenos Aires, Adriana Hidalgo Editora, 2008. Milner Max, L'envers du visible : essai sur l'ombre, Paris, Le Seuil, 2005.

Pline, Histoire naturelle Livre XXXV, Paris, trad. du latin J. M. Croisille, Les belles lettres, coll. « Classiques en poche », 1997. 
Popper Frank, Art, action et participation. L'artiste et la créativité aujourd'hui, Paris, Klincksieck, coll. « Esthétique », 1980.

Rancière Jacques, Le partage du sensible. Esthétique et politique, Paris, La Fabrique, 2000.

Rank Otto, Don Juan et le double, trad. de l'allemand S. Lautman, Paris, Payot et Rivages, coll. « Petite bibliothèque Payot », 2001.

Simondon Gilbert, Du mode d'existence des objets techniques, Paris, Aubier, coll. « L'invention philosophique », 1989.

Simondon Gilbert, Sur la technique, Paris, Puf, 2014.

Simondon Gilbert, L'individuation à la lumière des notions de forme et d'information, Grenoble, Millon, coll. « Krisis », 2013.

Szendy Peter, Le supermarché du visible. Essai d’iconomie, Paris, Minuit, coll. « Paradoxe », 2017.

Tackels Bruno, L'œuvre d'art à l'époque de W. Benjamin. Histoire d'aura, Paris, L'Harmattan, coll. « Esthétiques », 2000.

Torok Maria et Abraham Nicolás, L'écorce et le noyau, Paris, Flammarion, 1978.

Velter André, Ernest Pignon-Ernest, Paris, Gallimard, 2014.

\section{NOTES}

1. Jacques Rancière, Le partage du sensible. Esthétique et politique, Paris, La Fabrique, 2000.

2. On peut traduire Siluetazo comme « coup de silhouettes ", où « coup » a le même sens que dans « coup d'État ».

3. Ana Longoni et Gustavo Bruzzone (dir.), El siluetazo, Buenos Aires, Adriana Hidalgo Editora, 2008.

4. Ibid., p. 8.

5. Voir Max Milner, L'envers du visible : essai sur l'ombre, Paris, Le Seuil, 2005.

6. Voir Philippe Lacoue-Labarthe, "Portrait de l'artiste, en général ", in Écrits sur l'art, Genève, Les presses du réel, 2009, coll. « Mamco », p. 39-79.

7. Voir Otto Rank, Don Juan et le double, Paris, Payot et Rivages, coll. « Petite bibliothèque Payot », 2001. Jurgis Baltrušaitis, Le miroir: essai sur une légende scientifique. Révélations, science-fiction et fallacies, Paris, Le Seuil, 1978.

8. Voir Gilbert Simondon, Du mode d'existence des objets techniques, Paris, Aubier, coll. « L'invention philosophique », 1989, p. 181.

9. Voir Gilbert Simondon, Sur la technique, Paris, Puf, 2015.

10. Voir Gilbert Simondon, L'individuation à la lumière des notions de forme et d'information, Grenoble, Millon, coll. « Krisis », 2013.

11. Voir Gilbert Simondon, "L'effet de halo en matière technique: vers une stratégie de la publicité ", in Sur la technique, op. cit., p. 280-294.

12. Walter Benjamin, «L'œuvre d'art à l'époque de sa reproduction mécanisée ", in Écrits français [1933-1940], Paris, Gallimard, coll. « Folio essais », 2006, p. 149-248.

13. Walter Benjamin, Écrits français, op.cit., p. 190.

14. Jacques Derrida, «Freud et la scène de l'écriture ", in L'écriture et la différence, Paris, Minuit, p. 293.

15. Id.

16. Ibid., p. 294.

17. Ibid., p. 297. 
18. Ibid., p. 298.

19. Ibid., p. 299.

20. Id.

21. Siegmund Freud, "Esquisse d'une psychologie scientifique ", in La naissance de la psychanalyse, Paris, Puf, 1996.

22. Freud, dans la lettre qu'il adresse à Wilhelm Fliess le 20 novembre 1895 (il lui envoie l'Esquisse...) parle bien d'une «machine ». L'année suivante, dans une autre lettre, il explique au même destinataire les réajustements de la machine. Il y a bien au moins trois types d'inscriptions : «[...] les inscriptions individuelles sont séparées (de manière non nécessairement topique) selon porteurs neuroniques... Perception. Ce sont les neurones dans lesquels naissent les perceptions, auxquels se lie la conscience, mais qui ne gardent en eux-mêmes aucune trace de l'événement. Car la conscience et la mémoire s'excluent. C'est la première inscription des perceptions, tout à fait incapable d'accéder à la conscience, constituée par association simultanée. Inconscient. C'est la deuxième inscription... Préconscient. C'est la troisième inscription, liée aux représentations verbales, correspondant à notre moi officiel... » Cité par Jacques Derrida, «Freud et la scène de l'écriture ", in L'écriture et la différence, Paris, Minuit, p. 293, p. 306-307.

23. Ibid., p. 307.

24. Ibid., p. 310.

25. Ibid., p. 311.

26. Ibid., p. 314.

27. Id.

28. Ibid., p. 317.

29. Id.

30. Ibid., p. 334.

31. Ibid., p. 109.

32. Voir Georges Didi-Huberman, L'image survivante. Histoire de l'art et temps des fantômes selon Aby Warburg, Paris, Minuit, coll. « Paradoxe », 2002.

33. Jacques Derrida, Mal d'archive. Une impression freudienne, Paris, Galilée, coll. «Incises », 1995, p. 96.

34. «Le temps est hors de ses gonds ». C'est, comme on le sait, la phrase prononcée par Hamlet qui soutient tout le discours de Spectres de Marx.

35. Jacques Derrida, Mal d'archive, op.cit., p. 100.

36. Id.

37. Ibid., p. 128.

38. Ibid., p. 146.

39. Jacques Derrida, Mémoires d'aveugle. L'autoportrait et d'autres ruines, Paris, Réunion des Musées Nationaux, coll. « Parti pris », 1991, p. 54. Voir Pline, Histoire naturelle Livre XXXV, trad. du latin J.M. Croisille, Les belles lettres, Paris, 1997.

40. Ibid., p. 54.

41. Ibid., p. 56.

42. Ibid., p. 58.

43. Id.

44. Id.

45. Jean-Louis Déotte, L'époque de l'appareil perspectif. Brunelleschi, Machiavel, Descartes, Paris, L'Harmattan, coll. « Esthétiques », 2001.

46. Jean-Louis Déotte, Qu'est-ce qu'un appareil? Benjamin, Lyotard, Rancière, Paris, L'Harmattan, coll. « Esthétiques », 2007.

47. Jean-Louis Déotte, « La ligne d'horizon », Ici et ailleurs [en ligne], 8 juin 2011. URL : http://iciet-ailleurs.org/spip.php?article155 consulté le 15/09/2020.

48. Id. 
49. Voir Jean-Louis Déotte, L'époque des appareils, Paris, Lignes/Manifeste, 2004 ; Déotte JeanLouis, Walter Benjamin et la forme plastique. Architecture, technique, lieux, Paris, L'Harmattan, coll. « Esthétiques », 2012.

50. Voir Walter Benjamin, Paris, capitale du XIX siècle. Le livre des Passages [1939], Paris, Le Cerf, coll. « Passages », 1989.

51. Voir Konrad Fiedler, Sur l'origine de l'activité artistique, trad. de l'allemand I. Parvu et I. Rotermund, Paris, Rue d'Ulm, 2008; Konrad Fiedler, Essais sur l'art, Besançon, Les éditions de l'imprimeur, 2002.

52. Voir Asja Lācis, Walter Benjamin et le théâtre pour enfants prolétarien, trad. de l'allemand P. Ivernel, Paris, Le Portique, coll. « Carnets », 2007.

53. Bruno Tackels, L'œuvre d'art à l'époque de W. Benjamin. Histoire d'aura, Paris, L'Harmattan, coll. « Esthétiques », 2000, p. 76.

54. Voir Peter Szendy, Le supermarché du visible. Essai d'iconomie, Paris, Minuit, coll. « Paradoxe », 2017.

55. Même si Szendy, à la différence de Tackels, ne souligne pas la conclusion que Benjamin avait proposée, en ce qui concerne une irréductibilité - au moins en tant qu'idée - de l'appareil cinématographique par rapport aux marchandises (ce qu'Adorno et Horkheimer appelleront l'industrie culturelle); d'où la chance que le cinéma pourrait avoir de proposer une innervation émancipatrice. Szendy reste donc dans la conception adornienne selon laquelle il n'y a pas, pour le cinéma, un dehors des processus de marchandisation.

56. Pour lui, la seule manière d'interpréter le phénomène de l'innervation (et l'on dirait de tout phénomène social) est de le remettre à l'échangeabilité marchande. C'est une grille de lecture trop réductive du phénomène de la technique, comme le montre dans toute son œuvre Gilbert Simondon. Elle réduit aussi Benjamin à un pur épigone de Marx, ce qui (comme n'a cessé de le montrer Déotte dans ses livres sur Benjamin) ne fait pas justice a ce dernier.

57. On sait que c'est ici que se base la critique qu'Adorno adressera à Benjamin, puisque celui-là ne croit pas à une véritable dés-auratisation du cinéma; ou pour le dire avec Tackels, il ne comprend pas la thèse de la dés-auratisation chez Benjamin, laquelle n'implique pas la fin tout court de toute aura dans le cinéma.

58. Bruno Tackels, op. cit., p. 81.

59. Voir Frank Popper, Art, action et participation. L'artiste et la créativité aujourd'hui, Paris, Klincksieck, coll. « Esthétique », 1980.

60. Voir Nicolás Abraham et Maria Torok, L'écorce et le noyau, Paris, Flammarion, 1978.

61. André Velter, Ernest Pignon-Ernest, Paris, Gallimard, 2014, p. 84.

62. Id.

63. Une investigation judiciaire est toujours en cours à ce sujet, à la demande des proches du poète.

\section{RÉSUMÉS}

Dans cet article, nous nous proposons de développer une théorie philosophique de la trace qui pourra, à partir de la lecture que fait Jacques Derrida de la psychanalyse, élaborer une esthétique «spectrale » qui surgit de l'occupation des villes ayant dû faire face à des violences extrêmes (notamment la disparition politique) par des images des corps dans quelques expériences 
artistiques, comme celles du Siluetazo (1983) argentin. Pour ce faire, il nous faudra essayer d'établir les rapports entre trace, archive, inscription, images techniques et innervations des corps. Tout ceci nous conduira à montrer comment l'art, quand il dépasse ses limites institutionnelles et acquiert le statut d'expérience collective, est à même d'accueillir les fantômes qui hantent nos sociétés et nos villes en exigeant justice.

INDEX

Mots-clés : trace, spectralité, images techniques, innervation, crypte

\section{AUTEURS}

\section{ADOLFO VERA}

Docteur en philosophie, Université Paris 8 Vincennes - Saint-Denis, professeur à l'Institut de philosophie, Université de Valparaíso ; adolfo.vera@uv.cl 